\title{
The method of Puiseux series and invariant algebraic curves
}

\author{
Maria V. Demina \\ National Research University Higher School of Economics, 34 Tallinskaya Street, 123458, Moscow, \\ Russian Federation, maria_dem@mail.ru
}

\begin{abstract}
An explicit expression for the cofactor related to an irreducible invariant algebraic curve of a polynomial dynamical system in the plane is derived. A sufficient condition for a polynomial dynamical system in the plane to have a finite number of irreducible invariant algebraic curves is obtained. All these results are applied to Liénard dynamical systems $x_{t}=y, y_{t}=-f(x) y-g(x)$ with $\operatorname{deg} f<\operatorname{deg} g<2 \operatorname{deg} f+1$. The general structure of their irreducible invariant algebraic curves and cofactors is found. It is shown that Liénard dynamical systems with $\operatorname{deg} f<\operatorname{deg} g<2 \operatorname{deg} f+1$ can have at most two distinct irreducible invariant algebraic curves simultaneously and, consequently, are not integrable with a rational first integral.
\end{abstract}

Keywords: invariant algebraic curves, Darboux polynomials, Liénard systems

\section{Introduction}

The problem of finding invariant algebraic curves of polynomial dynamical systems in the plane has twofold interest. Firstly, invariant algebraic curves produce in explicit form certain phase curves of related dynamical systems. In particular, algebraic limit cycles are given by ovals of invariant algebraic curves. By an oval of an algebraic curve we mean a connected component lying in the projective plane $\mathbb{R} P^{2}$ and diffeomorphic to a circle. Secondly, the complete set of irreducible invariant algebraic curves is a key object in the study of Darboux and Liouvillian integrability of dynamical systems [1 [ $[$ ] .

Recently a novel method of finding invariant algebraic curves, which we shall call the method of Puiseux series, was introduced [7 9]. This method was used to establish the general structure of an irreducible invariant algebraic curve for any polynomial dynamical system in the plane [7]. In this article we present a further development of the method. We obtain some results concerning uniqueness of invariant algebraic curves. We derive a sufficient condition for a polynomial dynamical systems in the plane to have a finite number of irreducible invariant algebraic curves. Another novel results is an explicit expression for the cofactor related to an irreducible invariant algebraic curve. This expression is of importance in the theory of integrability. Indeed, investigating the linear dependence or independence of the cofactors related to irreducible invariant algebraic curves and exponential invariants enables one to solve the problem of Darboux integrability for a given dynamical system [1-5]. In addition, if the polynomial equal to the divergence of the vector field related to 
the dynamical system is added to the set of cofactors, then the Liouvillian integrability or non-integrability can be established [1 5$]$.

Further, we apply the method of Puiseux series to famous Liénard dynamical systems $x_{t}=y, y_{t}=-f(x) y-g(x)$ having a great number of physical applications. The integrability and limit cycles of Liénard dynamical systems have been intensively studied in recent years [7, 8, 10 20]. K. Odani was the first to show that Liénard dynamical systems with $\operatorname{deg} g \leq$ $\operatorname{deg} f$ do not have invariant algebraic curves excluding some trivial cases [10]. Liouvillian integrability of Liénard dynamical systems with $\operatorname{deg} g \leq \operatorname{deg} f$ was considered by J. Llibre and C. Valls [13, 17], see also [18]. The study of hyperelliptic limit cycles, i.e. limit cycles given by invariant algebraic curves of the form $(y+u(x))^{2}-v(x)=0$ with $u(x)$ and $v(x) \in \mathbb{R}[x]$, in Liénard dynamical systems was begun by $\mathrm{H}$. Żoładec [11] and was further continued by J. Llibre and X. Zhang [12], X. Yu and X. Zhang [14], C. Liu, G. Chen, and J. Yang [16].

In this article we consider Liénard dynamical systems with $\operatorname{deg} f<\operatorname{deg} g<2 \operatorname{deg} f+1$. In particular, we give the general structure of their irreducible invariant algebraic curves and corresponding cofactors. We prove that Liénard dynamical systems with $\operatorname{deg} f<\operatorname{deg} g<$ $2 \operatorname{deg} f+1$ can have at most two distinct irreducible invariant algebraic curves simultaneously. This fact means that Liénard dynamical systems in question are not integrable with a rational first integral. As an example, all irreducible invariant algebraic curves for a family of Liénard differential system with $\operatorname{deg} f=2$ and $\operatorname{deg} g=4$ are obtained. It turns out that there exist irreducible invariant algebraic curves of more complicated structure than it was supposed before. All these results seem to be new.

This article is organized as follows. In Section 2 we give a brief review of the method of Puiseux series and present novel results including the explicit expression for the cofactor of an irreducible invariant algebraic curve. In Section 3 we study Liénard dynamical systems with $\operatorname{deg} f<\operatorname{deg} g<2 \operatorname{deg} f+1$. In Section 4 we present the classification of irreducible invariant algebraic curves for a family of Liénard differential system with $\operatorname{deg} f=2$ and $\operatorname{deg} g=4$.

\section{The method of Puiseux series}

Let us consider the following dynamical system in the plane

$$
x_{t}=P(x, y), \quad y_{t}=Q(x, y), \quad P(x, y), \quad Q(x, y) \in \mathbb{C}[x, y] .
$$

By $\mathbb{C}[x, y]$ we denote the ring of polynomials in the variables $x$ and $y$ with coefficients in $\mathbb{C}$. The related polynomial vector field is defined as

$$
\mathcal{X}=P(x, y) \frac{\partial}{\partial x}+Q(x, y) \frac{\partial}{\partial y} .
$$

An algebraic curve $F(x, y)=0, F(x, y) \in \mathbb{C}[x, y] \backslash \mathbb{C}$ is an invariant algebraic curve of the vector field $\mathcal{X}$ if it satisfies the following equation $\mathcal{X} F=\lambda(x, y) F$, i.e.

$$
P(x, y) F_{x}+Q(x, y) F_{y}=\lambda(x, y) F
$$


where $\lambda(x, y) \in \mathbb{C}[x, y]$ is a polynomial called the cofactor of the invariant curve. It is straightforward to find that the polynomial $\lambda(x, y)$ is of degree at most $M-1$, where $M$ is the degree of $\mathcal{X}: M=\max \{\operatorname{deg} P, \operatorname{deg} Q\}$. For simplicity let us slightly abuse notation and call the polynomial $F(x, y)$ satisfying equation (2.3) an invariant algebraic curve bearing in mind that in fact the zero set of $F(x, y)$ is under consideration. Such a polynomial $F(x, y)$ giving an invariant algebraic curve is known as a Darboux polynomial. Note that we should take only one representative from the family $c F(x, y)$, where $c \in \mathbb{C}$ and $c \neq 0$.

Regarding the variable $y$ as dependent and the variable $x$ as independent, we find that the function $y(x)$ satisfies the following first-order algebraic ordinary differential equation

$$
P(x, y) y_{x}-Q(x, y)=0 .
$$

It is without loss of generality to suppose that the polynomials $P(x, y)$ and $Q(x, y)$ are relatively prime in $\mathbb{C}[x, y]$. Indeed, assuming the converse we shall only obtain a number of additional invariant algebraic curves given by the common zeros of the polynomials $P(x, y)$ and $Q(x, y)$. Solutions of equation (2.4) considered as curves in the plane can be viewed as a foliation. Rewriting (2.4) as the Pfaffian equation $\omega=0$, where $\omega=Q(x, y) d x-P(x, y) d y$ is the corresponding 1 -form, we see that the vector field $\mathcal{X}$ is tangent to the leaves of the foliation. Thus the problem of deriving irreducible invariant algebraic curves of the vector field $\mathcal{X}$ is equivalent to the problem of finding algebraic leaves of the corresponding foliation. For more details on the theory of polynomial foliations see [4].

Our aim is to describe an algebraic approach, which can be used to perform the classification of irreducible invariant algebraic curves. We say that an algebraic curve is irreducible if it is irreducible in the ring $\mathbb{C}[x, y]$. Note that extending the polynomial foliations from the affine plane $\mathbb{C}^{2}$ to the complex projective plane $\mathbb{C} P^{2}$, one can include into consideration the behavior of foliations at infinity. This extension can be used to obtain a number of powerful results, see [4]. Similarly, the major role in the method of Puiseux series is played by Puiseux series near infinity.

Let us recall some facts from the theory of fractional power or Puiseux series. A Puiseux series in a neighborhood of the point $x_{0}$ is defined as

$$
y(x)=\sum_{l=0}^{+\infty} b_{l}\left(x-x_{0}\right)^{\frac{l_{0}+l}{n_{0}}}
$$

where $l_{0} \in \mathbb{Z}, n_{0} \in \mathbb{N}$. In its turn a Puiseux series in a neighborhood of the point $x=\infty$ is given by

$$
y(x)=\sum_{l=0}^{+\infty} c_{l} x^{\frac{l_{0}-l}{n_{0}}}
$$

where again $l_{0} \in \mathbb{Z}, n_{0} \in \mathbb{N}$.

It is known [21] that a Puiseux series of the form (2.5) that satisfy equation $F(x, y)=0$ with $F(x, y) \in \mathbb{C}[x, y]$ is convergent in a neighborhood of the point $x_{0}$ (the point $x_{0}$ is excluded from domain of convergence if $\left.l_{0}<0\right)$. Analogously, a Puiseux series of the form (2.6) that satisfy equation $F(x, y)=0$ is convergent in a neighborhood of the point $x=\infty$ 
(the point $x=\infty$ is excluded from domain of convergence if $l_{0}>0$ ). If $n_{0}>1$ then the convergence of the corresponding series is understood in the sense that a certain branch of the $n_{0}$-th root is chosen and a cut forbidding going around the branch point is introduced.

The set of all Puiseux series of the form (2.5) or (2.6) forms a field, which we denote by $\mathbb{C}_{x_{0}}\{x\}$ or $\mathbb{C}_{\infty}\{x\}$ accordingly. In addition, we shall consider the rings of polynomials in one variable $y$ with coefficients from the fields $\mathbb{C}_{x_{0}}\{x\}$ or $\mathbb{C}_{\infty}\{x\}$. They will be referred to as $\mathbb{C}_{x_{0}}\{x\}[y]$ or $\mathbb{C}_{\infty}\{x\}[y]$ respectively.

It is straightforward to prove that invariant algebraic curves of the vector field $\mathcal{X}$ capture Puiseux series satisfying equation (2.4), [7]. All the Puiseux series that solve equation (2.4) can be obtained with the help of the power geometry and the Painlevé methods [22 25]. A brief description of such a method will be given in Appendix.

Let $W(x, y)$ be a formal sum of the monomials $c x^{q_{1}} y^{q_{2}}$ with bounded from above degrees, i.e. $q_{1}, q_{2} \in \mathbb{Q}, q_{1}+q_{2} \leq K$. Here $c$ as a constant from the field $\mathbb{C}$. We introduce the projection $\{W(x, y)\}_{+}$giving the sum of the monomials of $W(x, y)$ with integer nonnegative powers: $q_{1}, q_{2} \in \mathbb{N} \cup\{0\}$. In other words, $\{W(x, y)\}_{+}$yields the polynomial part of $W(x, y)$. It is straightforward to show that the projection is a linear operator.

The following theorem was proved in article [7].

Theorem 2.1. Let $F(x, y) \in \mathbb{C}[x, y] \backslash \mathbb{C}$ with $F_{y} \not \equiv 0$ be an irreducible invariant algebraic curve of the vector field $\mathcal{X}$ and related dynamical system (2.1). Then $F(x, y)$ takes the form

$$
F(x, y)=\left\{\mu(x) \prod_{j=1}^{N}\left\{y-y_{j}(x)\right\}\right\}_{+},
$$

where $\mu(x) \in \mathbb{C}[x]$, and $y_{1}(x), \ldots, y_{N}(x)$ are pairwise distinct Puiseux series in a neighborhood of the point $x=\infty$ that satisfy equation (2.4). Moreover, the degree of $F(x, y)$ with respect to $y$ does not exceed the number of distinct Puiseux series of the from (2.6) satisfying equation (2.4) whenever the latter is finite.

The first result of the present article concerns the cofactor of an irreducible invariant algebraic curve. Let us prove the following theorem.

Theorem 2.2. Let $F(x, y)$ given by (2.7) be an irreducible invariant algebraic curve of the vector field $\mathcal{X}$ and related dynamical system (2.1). Then the cofactor $\lambda(x, y)$ of $F(x, y)$ takes the form

$$
\lambda(x, y)=\left\{P(x, y) \sum_{m=0}^{\infty} \sum_{l=1}^{L} \frac{\nu_{l} x_{l}^{m}}{x^{m+1}}+\sum_{m=0}^{\infty} \sum_{j=1}^{N} \frac{\left\{Q(x, y)-P(x, y) y_{j, x}\right\} y_{j}^{m}}{y^{m+1}}\right\}_{+},
$$

where $x_{1}, \ldots, x_{L}$ are pairwise distinct zeros of the polynomial $\mu(x)$ with multiplicities $\nu_{1} \in \mathbb{N}$, $\ldots, \nu_{L} \in \mathbb{N}$ and $L \in \mathbb{N} \cup\{0\}$. If $\mu(x)=\mu_{0}$ where $\mu_{0} \in \mathbb{C}$, then we suppose that $L=0$ and the first series is absent in (2.8). 
Proof. We begin the proof representing the irreducible invariant algebraic curve $F(x, y)$ in the ring $\mathbb{C}_{\infty}\{x\}[y]$ as follows

$$
F(x, y)=\mu(x) \prod_{j=1}^{N}\left\{y-y_{j}(x)\right\}
$$

The polynomial $\mu(x)$ takes the form

$$
\mu(x)=\prod_{l=1}^{L}\left(x-x_{l}\right)^{\nu_{l}}, \quad \nu_{l} \in \mathbb{N}, \quad L \in \mathbb{N} \cup\{0\} .
$$

If $\mu(x)=\mu_{0}$ where $\mu_{0} \in \mathbb{C}$, then we set $L=0$. From (2.9) it follows that the correlations

$$
\frac{F_{x}}{F}=\frac{\mu_{x}}{\mu}-\sum_{j=1}^{N} \frac{y_{j, x}}{y-y_{j}} ; \quad \frac{F_{y}}{F}=\sum_{j=1}^{N} \frac{1}{y-y_{j}}
$$

are valid. Further, we find the Laurent series of the functions $F_{x} / F$ and $F_{y} / F$ near the point $y=\infty$. Substituting the resulting expressions into (2.3), we get

$$
\lambda(x, y)=P(x, y) \sum_{m=0}^{\infty} \sum_{l=1}^{L} \frac{\nu_{l} x_{l}^{m}}{x^{m+1}}+\sum_{m=0}^{\infty} \sum_{j=1}^{N} \frac{\left\{Q(x, y)-P(x, y) y_{j, x}\right\} y_{j}^{m}}{y^{m+1}}
$$

where the function

$$
\frac{\mu_{x}}{\mu}=\sum_{l=1}^{L} \frac{\nu_{l}}{x-x_{l}}
$$

is also expanded into the Laurent series near the point $x=\infty$ Finally, we recall that $\lambda(x, y)$ is a bivariate polynomial. This yields (2.8).

Note that the roles of $x$ and $y$ in Theorems 2.1 and 2.2 can be changed. In addition, representations (2.7) and (2.8) for an invariant algebraic curve and its cofactor are still valid if the requirement of irreducibility is removed. But in such a case there may arise coinciding Puiseux series in these representations.

Now let us show that the degree of the polynomial $\mu(x)$, which is the coefficient at the highest-order term $y^{N}$ in the explicit expression of $F(x, y)$, can be deduced with the help of Puiseux series of the form (2.5) that satisfy equation (2.4).

Lemma 2.1. Let $F(x, y) \in \mathbb{C}[x, y] \backslash \mathbb{C}$ with $F_{y} \not \equiv 0$ be an irreducible invariant algebraic curve of the vector field $\mathcal{X}$ and related dynamical system (2.1). If $x_{0} \in \mathbb{C}$ is a zero of the polynomial $\mu(x)$, then at least one Puiseux series of the form (2.5) with $l_{0}<0$ satisfies equation (2.4). 
Proof. Considering the field $\mathbb{C}_{x_{0}}\{x\}$, which is algebraically closed [21], we see that there exists uniquely determined system of elements $y_{j, x_{0}}(x) \in \mathbb{C}_{x_{0}}\{x\}$ such that the following representation is valid [21]

$$
F(x, y)=\mu(x) \prod_{j=1}^{N}\left\{y-y_{j, x_{0}}(x)\right\},
$$

where $N$ as before is the degree of $F(x, y)$ with respect to $y$. The set of elements $y_{j, x_{0}}(x) \in$ $\mathbb{C}_{x_{0}}\{x\}$ appearing in representation (2.14) is a subset of those satisfying equation (2.4), see [7]. Let us assume that all the Puiseux series of the form (2.5) that satisfy equation (2.4) have $l_{0} \geq 0$. Thus all the series in representation (2.14) also satisfy this condition. Since $x_{0}$ is a zero of $\mu(x)$, we see that $F(x, y)$ given by (2.14) is reducible. It is a contradiction. Consequently, at least one Puiseux series near the point $x_{0}$ with negative exponent at the leading-order term satisfies equation (2.4).

Consequence. If for all $x_{0} \in \mathbb{C}$ the Puiseux series of the form (2.5) satisfying equation (2.4) have non-negative exponents at the leading-order terms, then $\mu(x)=\mu_{0}$ where $\mu_{0}$ is a constant. Without loss of generality, one can set $\mu_{0}=1$.

Lemma 2.2. Suppose there exists finite number of Puiseux series of the form (2.5) that satisfy equation (2.4) and have negative exponents at the leading-order terms:

$$
\begin{gathered}
y_{j, x_{0}}(x)=b_{0}^{(j)}\left(x-x_{0}\right)^{-q_{j}}+\ldots, \quad b_{0}^{(j)} \neq 0, \\
q_{j} \in \mathbb{Q}, \quad q_{j}>0, \quad 1 \leq j \leq J \in \mathbb{N} .
\end{gathered}
$$

If $F(x, y)$ is an irreducible invariant algebraic curve of the vector field $\mathcal{X}$ and related $d y$ namical system (2.1), then the multiplicity $\nu_{0} \in \mathbb{N}$ of the zero of the polynomial $\mu(x)$ at the point $x_{0}$ satisfies the inequality

$$
\nu_{0} \leq \sum_{j=1}^{J} q_{j}
$$

Proof. Again we consider the representation of $F(x, y)$ in the ring $\mathbb{C}_{x_{0}}\{x\}[y]$, which is given by (2.14). The Puiseux series appearing in this representation satisfy equation (2.4), see [7]. Rewriting the polynomial $\mu(x)$ as $\mu(x)=\mu_{0}\left(x-x_{0}\right)^{\nu_{0}}+\ldots$, where $\mu_{0} \neq 0$, we see that the factor $\left(x-x_{0}\right)^{\nu_{0}}$ should compensate the terms with negative exponents in such a way that the resulting expression is an irreducible polynomial. The multiplicity of $\mu(x)$ at its zero $x_{0}$ is the highest if all the series given by (2.15) are captured by representation (2.14) and other Puiseux series in (2.14) have non-zero coefficients at $\left(x-x_{0}\right)^{0}$. Since $F(x, y)$ is irreducible, we see that each series $y_{j, x_{0}}(x)$ given by (2.15) can enter representation (2.14) at most once. Assuming that condition (2.16) is not satisfied, we come to a contradiction. Indeed, the polynomial $F(x, y)$ given by (2.14) is reducible whenever condition (2.16) is not satisfied. This completes the proof. 
Now we shall study uniqueness properties of irreducible invariant algebraic curves of the vector field $\mathcal{X}$.

Theorem 2.3. Suppose a Puiseux series $y(x)$ of the form (2.5) or (2.6) with uniquely determined coefficients satisfies equation (2.4); then there exists at most one irreducible invariant algebraic curve $F(x, y)$ of the vector field $\mathcal{X}$ and related dynamical system (2.1) such that this series is captured by $F(x, y)$, i.e. $F(x, y(x))=0$.

Proof. The proof is by contradiction. Let us suppose that the vector field $\mathcal{X}$ and related dynamical system (2.1) possess at least two distinct irreducible invariant algebraic curves given by the polynomials $F_{1}(x, y)$ and $F_{2}(x, y)$ such that $F_{1}(x, y(x))=0$ and $F_{2}(x, y(x))=0$. We see that these two curves intersect in an infinite number of points inside the domain of convergence of the series $y(x)$. It follows from the Bézout's theorem that there exists a polynomial both dividing $F_{1}(x, y)$ and $F_{2}(x, y)$. The polynomial $F_{1}(x, y)$ is irreducible. Thus we conclude that $F_{2}(x, y)=h(x, y) F_{1}(x, y)$, where $h(x, y) \in \mathbb{C}[x, y]$. But the polynomial $F_{2}(x, y)$ is also irreducible. Consequently, $h \in \mathbb{C}$ and the polynomials $F_{1}(x, y)$ and $F_{2}(x, y)$ define the same curve. It is a contradiction.

Theorem 2.4. If the number of Puiseux series of the form (2.6) that satisfy equation (2.4) is finite, then the vector field $\mathcal{X}$ and related dynamical system (2.1) possess a finite number (possibly none) of irreducible invariant algebraic curves and, consequently, cannot have a rational first integral. Moreover, the number of distinct irreducible invariant algebraic curves $F(x, y)$ such that $F_{y} \not \equiv 0$ does not exceed the number of distinct Puiseux series of the form (2.6) that satisfy equation (2.4).

Proof. It is straightforward to show that the number of irreducible invariant algebraic curves that do not depend on $y$ is finite. Indeed, substituting $F=x-x_{0}, x_{0} \in \mathbb{C}$ into equation (2.3), we get $P(x, y)=\lambda(x, y)\left(x-x_{0}\right)$. It follows from this relation that there may exist only a finite number of admissible values of the parameter $x_{0}$.

Further, recalling the Newton-Puiseux algorithm of finding Puiseux series in explicit form [21], we see that polynomials $F(x, y)$ representing irreducible invariant algebraic curves in the case $F_{y} \not \equiv 0$ cannot involve an arbitrary parameter. Indeed, if such a parameter existed, then it would enter Puiseux series appearing in representation (2.7). But all these series satisfy equation (2.4) and have no arbitrary parameters according to the statement of the theorem. Note that we do not take into consideration the parameter $c$ resulting from the equivalence $F \sim c F$. It follows from the previous observations and Theorem 2.3 that the number of distinct irreducible invariant algebraic curves is finite. This number is the highest if all the Puiseux series of the form (2.6) satisfying equation (2.4) are Laurent series that terminate at zero term. In such a case this number is equal to the number of distinct Puiseux series of the form (2.6) that satisfy equation (2.4). Finally, supposing that the vector field $\mathcal{X}$ and related dynamical system (2.1) are integrable with a rational first integral, we come to a contradiction. Indeed, in such a case all (not some) integral curves are algebraic. 
Remark. Similar theorem is valid if Puiseux series of the form (2.5) that satisfy equation (2.4) are considered.

Concluding this section let us present the resulting description of the method of Puiseux series.

At the first step one should find all the Puiseux series (near finite points and infinity) that satisfy equation (2.4).

At the second step one uses Theorem 2.1 and Lemmas 2.1, 2.2 in order to derive the structure of an irreducible invariant algebraic curve and its cofactor, see relations (2.7) and (2.8). Note that at this step all possible combinations of Puiseux series near infinity found at the first step should be considered if one wishes to classify irreducible invariant algebraic curves. Requiring that the non-polynomial part of expression in brackets in (2.7) vanishes, one obtains a system of algebraic equations. This system contains infinite number of equation. In practice one takes several first equations. Note that equations resulting from the fact that the non-polynomial part of expression in brackets in (2.8) should vanish can be also considered.

At the third step one solves the algebraic system and makes the verification substituting the resulting irreducible invariant algebraic curve and its cofactor into equation (2.3).

Remark 1. In the above, we have considered irreducible invariant algebraic curves $F(x, y)$ such that $F_{y}(x, y) \not \equiv 0$. Irreducible invariant algebraic curves of the form $F=F(x)$ can be found substituting expression $F=x-x_{0}$ with $x_{0} \in \mathbb{C}$ into equation (2.3) and setting to zero the coefficients at different powers of $y$.

Remark 2. If the polynomial vector field under consideration possesses a rational first integral, then there exist infinite number of irreducible invariant algebraic curves. In such a situation one needs to construct the irreducible invariant algebraic curves $F_{1}(x, y), \ldots$, $F_{K}(x, y)$ such that their cofactors $\lambda_{1}(x, y), \ldots, \lambda_{K}(x, y)$ satisfy the following condition [1]

$$
d_{1} \lambda_{1}+\ldots+d_{K} \lambda_{K}=0, \quad d_{1}, \ldots, d_{K} \in \mathbb{Z} \backslash\{0\}, \quad K \in \mathbb{N} .
$$

All other irreducible invariant algebraic curves are expressible via $F_{1}(x, y), \ldots, F_{K}(x, y)$. Recall that a rational function $R(x, y) \in \mathbb{C}(x, y) \backslash \mathbb{C}$ is a rational first integral of the vector field $\mathcal{X}$ if $\mathcal{X} R=0$. For another method of finding rational first integrals, which uses Taylor and Puiseux series, see the work by A. Ferragut and H. Giacomini [26].

An example of the method of Puiseux series in practice will be given in Section 4 .

\section{Invariant algebraic curves for Liénard dynamical systems}

The polynomial dynamical systems of the form

$$
x_{t}=y, \quad y_{t}=-f(x) y-g(x)
$$

are known as Liénard dynamical systems. In this article we suppose that $f(x)$ and $g(x)$ are polynomials

$$
f(x)=f_{0} x^{m}+\ldots+f_{m}, \quad g(x)=g_{0} x^{n}+\ldots+g_{n}, \quad f_{0} g_{0} \neq 0
$$


with coefficients in the field $\mathbb{C}$. In addition we set $m<n<2 m+1$. The case $n=m+1$ was considered in [8, 20].

Let us prove the following theorem.

Theorem 3.1. Let $F(x, y) \in \mathbb{C}[x, y] \backslash \mathbb{C}$ be an irreducible invariant algebraic curve of Liénard dynamical system (3.1) with $\operatorname{deg} f<\operatorname{deg} g<2 \operatorname{deg} f+1$. Then $F(x, y)$ and its cofactor take the form

$$
\begin{gathered}
F(x, y)=\left\{\prod_{j=1}^{N-k}\left\{y-y_{j}(x)\right\}\left\{y-y_{N}(x)\right\}^{k}\right\}_{+}, \\
\lambda(x, y)=-N f-(N-k) q_{x}-k p_{x},
\end{gathered}
$$

where $k=0$ or $k=1, N \in \mathbb{N}$, and $y_{1}(x), \ldots, y_{N}(x)$ are the series

$$
\begin{aligned}
& (I): y_{j}(x)=q(x)+\sum_{l=0}^{\infty} c_{m+1+l}^{(j)} x^{-l}, \quad j=1, \ldots, N-k \\
& (I I): y_{N}(x)=p(x)+\sum_{l=0}^{\infty} c_{n-m+l}^{(N)} x^{-l} .
\end{aligned}
$$

The coefficients of the series of type (II) and of the polynomials

$$
\begin{gathered}
q(x)=-\frac{f_{0}}{m+1} x^{m+1}+\sum_{l=1}^{m} q_{m+1-l} x^{l} \in \mathbb{C}[x], \\
p(x)=-\frac{g_{0}}{f_{0}} x^{n-m}+\sum_{l=1}^{n-m-1} p_{n-m-l} x^{l} \in \mathbb{C}[x]
\end{gathered}
$$

are uniquely determined. The coefficients $c_{m+1}^{(j)}, j=1, \ldots, N-k$ are pairwise distinct. All other coefficients $c_{m+1+l}^{(j)}, l \in \mathbb{N}$ are expressible via $c_{m+1}^{(j)}$, where $j=1, \ldots, N-k$. The corresponding product in (3.3) is unit whenever $k=1$ and $N=1$.

Proof. Invariant algebraic curves of dynamical system (3.1) satisfy the following equation

$$
y F_{x}-\{f(x) y+g(x)\} F_{y}=\lambda(x, y) F .
$$

Substituting $F=F(x)$ into this equation, we verify that there are no invariant algebraic curves that do not depend on $y$.

Let $F(x, y)$ with $F_{y} \not \equiv 0$ be an irreducible invariant algebraic curve of dynamical system (3.1). Equation (2.4) now takes the form

$$
y y_{x}+f(x) y+g(x)=0 .
$$

For all $x_{0} \in \mathbb{C}$ the Puiseux series of the form (2.5) satisfying equation (3.8) have nonnegative exponents at the leading-order terms. Using Lemma 2.1, we find $\mu(x)=\mu_{0}$, where $\mu_{0}$ is a constant. Without loss of generality, we set $\mu_{0}=1$. 
There exist only two dominant balances that produce Puiseux series in a neighborhood of the point $x=\infty$. The equations related to these balances and their solutions are the following

$$
\begin{gathered}
(I): \quad y\left(y_{x}+f_{0} x^{m}\right)=0, \quad y(x)=-\frac{f_{0}}{m+1} x^{m+1} \\
(I I): \quad x^{m}\left(f_{0} y+g_{0} x^{n-m}\right)=0, \quad y(x)=-\frac{g_{0}}{f_{0}} x^{n-m} .
\end{gathered}
$$

In the case $(I)$ the corresponding Puiseux series have one arbitrary coefficient at $x^{0}$ provided that the compatibility condition related to the unique Fuchs index $l_{0}=m+1$ is satisfied. Let us recall that the definition of Fuchs indices depends on the numeration of the series under consideration. Definitions of dominant balances and Fuchs indices can be found in Appendix, see also [7, 22 25]. In this article we use the numeration for all the series as given in (2.5) and (2.6). If the parameters of system (3.1) are chosen in such a way that the compatibility condition is not satisfied, then the series of type $(I)$ does not exist. All the coefficients of the series of type $(I I)$ are uniquely determined. Note that the Puiseux series are in fact Laurent series, they are given in (3.5). Factoring $F(x, y)$ in the ring $\mathbb{C}_{\infty}\{x\}[y]$ and taking the polynomial part of this factorization, we obtain (3.3). Since the polynomial $F(x, y)$ in (3.3) is irreducible, we conclude that the series $y_{1}(x), \ldots, y_{N-k}(x)$ should be pairwise distinct, see Theorem 2.1. Thus the irreducibility of $F(x, y)$ requires the coefficients $c_{m+1}^{(j)}, j=1, \ldots, N-k$ to be pairwise distinct and $k=0$ or $k=1$.

Finally, substituting $L=0(\mu(x)=1)$ and series (3.5) into expression (2.8), we find the cofactor as given in (3.4). This completes the prove.

Remark. If the parameters of system (3.1) are chosen in such a way that the compatibility condition for the series of type $(I)$ is not satisfied, then the unique irreducible invariant algebraic curve takes the form $F(x, y)=y-p(x)-c_{n-m}^{(N)}$ provided that the series of type $(I I)$ terminates at zero term.

Further, our aim is to show that there exist a dynamical system equivalent to Liénard dynamical system (3.1) such that the algebraic ordinary differential equation related to this new system possesses finite number of Puiseux series near infinity with respect to one of the variables. Let us introduce the invertible change of variables $x=s, y=z+q(s) \leftrightarrow s=x$, $z=y-q(x)$. The polynomial $q(x)$ is given in (3.6). Substituting the series of type $(I)$ into equation (3.8), we find the following recurrence relation

$$
q_{l}=\frac{(m+1)}{(m-l+1) f_{0}}\left\{\sum_{k=0}^{l-1} q_{k} f_{l-k}+\sum_{k=1}^{l-1}(m+1-k) q_{k} q_{l-k}+g_{n+l-(2 m+1)}\right\},
$$

where $1 \leq l \leq m, q_{0}=-f_{0} /(m+1), g_{-l}=0, l \in \mathbb{N}$ and the second sum equals zero in the case $l=1$. The new dynamical system takes the form

$$
s_{t}=z+q(s), \quad z_{t}=-\left\{q_{s}(s)+f(s)\right\}\{z+q(s)\}-g(s) .
$$


There exists the one-to-one correspondence between irreducible invariant algebraic curves $F(x, y)$ of Liénard dynamical system (3.1) and irreducible invariant algebraic curves $G(s, z)$ of system (3.11).

Theorem 3.2. Suppose $G(s, z) \in \mathbb{C}[s, z] \backslash \mathbb{C}$ is an irreducible invariant algebraic curve of dynamical system (3.11), where the coefficients of the polynomial $q(s)$ are given by (3.10). Then the degree of $G(s, z)$ with respect to $s$ is either 0 or $m+1$.

Proof. Let $G(s, z)$ be an irreducible invariant algebraic curve of dynamical system (3.11). In what follows we regard $s$ as a dependent variable and $z$ as an independent. Our aim is to find the representation of $G(s, z)$ in $\mathbb{C}_{\infty}\{z\}[s]$. The equation for the function $s(z)$ reads as

$$
\left[\left\{q_{s}(s)+f(s)\right\} z+\left\{q_{s}(s)+f(s)\right\} q(s)+g(s)\right] s_{z}+q(s)+z=0 .
$$

Using the definition of the polynomials $f, g$, and $q$, we obtain the following relations

$$
\begin{gathered}
\operatorname{deg}\left(\left\{q_{s}(s)+f(s)\right\} q(s)+g(s)\right) \leq m, \\
\operatorname{deg}\left(q_{s}(s)+f(s)\right) \leq m-1, \operatorname{deg} q(s)=m+1 .
\end{gathered}
$$

It follows from these relations that equation (3.12) possesses only one dominant balance producing Puiseux series in a neighborhood of the point $z=\infty$. The equation related to this balance and its solutions take the form

$$
f_{0} s^{m+1}-(m+1) z=0, \quad s^{(k)}(z)=b_{0}^{(k)} z^{1 /(m+1)}, \quad k=1, \ldots, m+1,
$$

where $b_{0}^{(1)}, \ldots, b_{0}^{(m+1)}$ are distinct roots of the equation $f_{0} b_{0}^{m+1}-(m+1)=0$. The balance (3.14) is algebraic. We find $m+1$ distinct Puiseux series in a neighborhood of the point $z=\infty$. The representation of $G(s, z)$ in the ring $\mathbb{C}_{\infty}\{z\}[s]$ is the following

$$
G(s, z)=\nu(z) \prod_{k=1}^{m+1}\left\{s-b_{0}^{(k)} z^{1 /(m+1)}-\ldots\right\}^{n_{k}},
$$

where $\nu(z) \in \mathbb{C}[z]$ and $n_{k}=0$ or $n_{k}=1$. Since $G(s, z)$ should be a polynomial, we obtain from representation (3.15) that the degree of $G(s, z)$ with respect to $s$ is either $0\left(n_{k}=0\right.$, $k=1, \ldots, m+1)$ or $m+1\left(n_{k}=1, k=1, \ldots, m+1\right)$.

The latter theorem is very important for applications, because the bound on the degrees of irreducible invariant algebraic curves established in this theorem can be used to find all irreducible invariant algebraic curves of systems (3.1) and (3.11) in explicit form. It follows from Theorem 2.4 that the number of distinct irreducible invariant algebraic curves of dynamical system (3.11) is finite. The same is true for Liénard dynamical systems (3.1), see Theorem 3.3 below.

The change of variables $x=s, y=z+q(s) \leftrightarrow s=x, z=y-q(x)$ allows us to make a refinement in Theorem 3.1 . 
Lemma 3.1. Suppose we are in assumptions of Theorem 3.1. If an irreducible invariant algebraic curve of Liénard dynamical system (3.1) with $\operatorname{deg} f<\operatorname{deg} g<2 \operatorname{deg} f+1$ does not capture the Puiseux series of type (II), then the polynomial $F(x, y)$ is of first degree with respect to $y$.

Proof. Any irreducible invariant algebraic curve $F(x, y)$ given by (3.3) with $k=0$ corresponds to an irreducible invariant algebraic curve $G(s, z)$ of dynamical system (3.11) such that the polynomial $G(s, z)$ does not depend on $s$. The latter, if exists, takes the form $G(s, z)=z-z_{0}$ with $z_{0} \in \mathbb{C}$. Consequently, irreducible invariant algebraic curves of dynamical system (3.1), if any, are of the form $F(x, y)=y-q(x)-z_{0}$ provided that $k=0$.

Theorem 3.3. Liénard dynamical systems (3.1) with $\operatorname{deg} f<\operatorname{deg} g<2 \operatorname{deg} f+1$ and fixed coefficients of the polynomials $f(x)$ and $g(x)$ have at most two distinct irreducible invariant algebraic curves simultaneously.

Proof. First of all we recall that Liénard dynamical systems (3.1) have no invariant algebraic curves that do not depend on $y$. Further, it follows from Theorem 2.3 that there exists at most one irreducible invariant algebraic curve with $k=1$ in representation (3.3).

Using Lemma 3.1, we see that if $k=0$ in representation (3.3), then $N=1$. In this case irreducible invariant algebraic curves, if any, can be written as $F(x, y)=y-q(x)-z_{0}$. If such curves exist, then algebraic ordinary differential equation (3.8) should have a polynomial solution of the form $y(x)=q(x)+z_{0}$. Substituting this function into the equation in question and using the fact that the coefficients of the polynomials $f(x), g(x)$, and $q(x)$ are uniquely determined, we find the unique value $z_{0}$. The same result can be obtained if we recall that invariant algebraic curves in the case $k=0$ and $N=1$ can be obtained requiring that the series of type $(I)$ terminates at zero term. Analyzing the recurrence relation for the coefficients of this series, which is similar to (3.10), we see that the first appearance of $c_{m+1}$ in the expressions for $c_{m+1+l}, l \in \mathbb{N}$ is with non-zero coefficient and unit exponent.

Let us sum up our results: if the two distinct irreducible invariant algebraic curves exist, then the first has $k=1$ in representation (3.3) and the second is of first degree with respect to $y$ and takes the form $F(x, y)=y-q(x)-z_{0}$.

Consequence. Liénard dynamical systems (3.1) with $\operatorname{deg} f<\operatorname{deg} g<2 \operatorname{deg} f+1$ are not integrable with a rational first integral.

\section{Examples}

As an example, we shall consider the Liénard dynamical system of type $\operatorname{deg} f=2$, $\operatorname{deg} g=4$ :

$$
x_{t}=y, \quad y_{t}=-\left(\zeta x^{2}+\beta x+\alpha\right) y-\left(\varepsilon x^{4}+\xi x^{3}+e x^{2}+\sigma x+\delta\right), \quad \zeta \varepsilon \neq 0 .
$$

The change of variables $x \mapsto X\left(x+x_{0}\right), y \mapsto Y y, T \mapsto T t, X Y T \neq 0$ relates system (4.1) with its simplified version at $\zeta=3, \varepsilon=-3$, and $\beta=0$. Thus without loss of generality, we 


\begin{tabular}{|c|c|c|}
\hline Invariant algebraic curves & Cofactors & Parameters \\
\hline $\begin{array}{l}y-x^{2}+\frac{1}{3}(\alpha+e) \\
y+x^{3}+\frac{3}{2} x^{2}+\left(\alpha-\frac{5}{2}\right) x+\frac{5 \alpha}{6}-\frac{\sigma}{3}-\frac{25}{12} \\
y^{2}+\left(x^{3}+\frac{1}{2} x^{2}-\frac{37}{4} x+\frac{35}{8}\right) y-x^{5}-\frac{3}{2} x^{4} \\
+\frac{35}{2} x^{3}+\frac{37}{4} x^{2}-\frac{1365}{16} x+\frac{1225}{32} \\
y^{2}+\left(x^{3}+\frac{1}{2} x^{2}+\frac{11}{4} x+\frac{51}{8}\right) y-x^{5}-\frac{3}{2} x^{4} \\
-\frac{5}{2} x^{3}-\frac{35}{4} x^{2}-\frac{69}{16} x+\frac{153}{32} \\
y^{2}+\left(x^{3}+\frac{1}{2} x^{2}-\frac{95}{12} x+\frac{1505}{216}\right) y-x^{5} \\
-\frac{3}{2} x^{4}+\frac{275}{18} x^{3}+\frac{335}{108} x^{2}-\frac{31175}{432} x+\frac{508475}{7776} \\
y^{3}+\left(2 x^{3}+2 x^{2}-\frac{1}{2} x+\frac{35}{2}\right) y^{2}+\left(x^{6}+x^{5}\right. \\
\left.-\frac{5}{4} x^{4}+\frac{39}{2} x^{3}+\frac{103}{16} x^{2}-\frac{335}{16} x+\frac{7325}{64}\right) y \\
-x^{8}-3 x^{7}+\frac{1}{2} x^{6}-\frac{63}{4} x^{5}-47 x^{4}+\frac{519}{16} x^{3} \\
-\frac{2065}{32} x^{2}-\frac{16325}{64} x+\frac{36625}{256}\end{array}$ & $\begin{array}{c}-3 x^{2}-2 x-\alpha \\
3 x-\frac{5}{2} \\
-3 x^{2}+x+\frac{17}{4} \\
-3 x^{2}+x-\frac{31}{4} \\
-3 x^{2}+x+\frac{35}{12} \\
-3 x^{2}+4 x-\frac{29}{4}\end{array}$ & $\begin{array}{l}\delta=\frac{\alpha(e+\alpha)}{3} \\
\sigma=\frac{2(e+\alpha)}{3} \\
\delta=\frac{25 \alpha}{12}-\frac{5 \sigma}{6}-\frac{125}{24}, \\
e=\frac{45}{4}-3 \alpha \\
\alpha=-\frac{27}{4}, e=\frac{63}{2}, \\
\delta=-\frac{595}{16}, \sigma=-\frac{9}{2} \\
\alpha=\frac{21}{4}, e=-\frac{9}{2}, \\
\delta=\frac{93}{16}, \sigma=-\frac{17}{2} \\
\alpha=-\frac{65}{12}, e=\frac{55}{2}, \\
\delta=-\frac{11825}{432} \\
\sigma=-\frac{185}{18} \\
\alpha=\frac{9}{4}, e=\frac{9}{2}, \\
\delta=\frac{145}{16}, \sigma=-\frac{39}{2}\end{array}$ \\
\hline $\begin{array}{l}y^{2}+\left(x^{3}+\frac{1}{2} x^{2}+\left\{\alpha-\frac{5}{2}\right\} x+\frac{5}{6}\{5\right. \\
\left.\left.-\frac{1}{27} \alpha\right\}\right) y-x^{5}-\frac{3}{2} x^{4}+\frac{5}{12}\{15-4 \alpha\} x^{3} \\
+\left\{\frac{35}{24}-\frac{187}{162} \alpha\right\} x^{2}+\left\{\frac{11327}{14580} \alpha-\frac{353}{108}\right\} x \\
+\frac{131545}{34992}-\frac{93553}{472392} \alpha\end{array}$ & $\begin{array}{c}-3 x^{2}+x \\
-\alpha-\frac{5}{2}\end{array}$ & $\begin{array}{l}\alpha^{2}-\frac{4975}{486} \alpha+\frac{4225}{144} \\
=0, e=\frac{45}{4}-3 \alpha \\
\delta=\frac{5101 \alpha}{14580}+\frac{193}{54} \\
\sigma=\frac{\alpha}{9}-\frac{15}{4}\end{array}$ \\
\hline
\end{tabular}

Table 1: Irreducible invariant algebraic curves of dynamical system (4.2) with $\xi=-2$. The curves in the last row have complex-valued coefficients provided that the coefficients of the original system are from $\mathbb{R}$.

obtain the dynamical system

$$
x_{t}=y, \quad y_{t}=-\left(3 x^{2}+\alpha\right) y+\left(3 x^{4}-\xi x^{3}-e x^{2}-\sigma x-\delta\right) .
$$

The complete set of irreducible invariant algebraic curves for dynamical system (4.2) is rather cumbersome. We shall present results for the dynamical systems with $\xi=-2$. We have chosen this family because there exist irreducible invariant algebraic curves of degrees (with respect to $y$ ) higher than 2. It seems that the classification of irreducible invariant algebraic curves for families of Liénard dynamical system of type $\operatorname{deg} f=2 \operatorname{deg} g=4$ is presented here for the first time.

Theorem 4.1. There unique irreducible invariant algebraic curves of Liénard dynamical system (4.2) with $\xi=-2$ are those given in Table 1 .

Proof. While proving this theorem we shall use results of Theorem 3.1 and Lemma 3.1. The 
Puiseux series given in (3.5) are now the following

$$
\begin{aligned}
& (I): \quad y(x)=-x^{3}-\frac{3}{2} x^{2}+\left(\xi-\alpha+\frac{9}{2}\right) x+c_{3}+\sum_{l=1}^{\infty} c_{l+3} x^{-l} \\
& (I I): \quad y(x)=x^{2}-\frac{1}{3}(\xi+2) x+\frac{1}{3}(\xi+2-\alpha-e)+\sum_{l=1}^{\infty} a_{l+2} x^{-l} .
\end{aligned}
$$

The Puiseux series $(I)$ has an arbitrary coefficient $c_{3}$ and exists whenever $e=3(27+6 \xi-$ $4 \alpha) / 4$. The Puiseux series $(I I)$ possesses uniquely determined coefficients. Note that we use novel designations for the coefficients of the Puiseux series $(I I)$. The general structure of irreducible invariant algebraic curves and their cofactors take the form

$$
\begin{gathered}
F(x, y)=\left\{\prod_{j=1}^{N-k}\left\{y+x^{3}+\frac{3}{2} x^{2}-\left(\xi-\alpha+\frac{9}{2}\right) x-c_{3}^{(j)}-\ldots\right\}\right. \\
\left.\times\left\{y-x^{2}-\ldots\right\}^{k}\right\}_{+} \\
\lambda(x, y)=-3 k x^{2}+(3 N-5 k) x+\frac{1}{6}(8 \xi+31-6 \alpha) k-\frac{1}{2}(2 \xi+9) N,
\end{gathered}
$$

where $k=0$ or $k=1, N \in \mathbb{N}$. If $e \neq 3(27+6 \xi-4 \alpha) / 4$, then it follows from Theorem 3.1 that the dynamical system under consideration possesses only one irreducible invariant algebraic curve

$$
\begin{gathered}
F(x, y)=y-x^{2}+\frac{1}{3}(\xi+2) x-\frac{1}{3}(\xi+2-\alpha-e), \\
\lambda(x, y)=-3 x^{2}-2 x-\alpha+\frac{1}{3}(\xi+2)
\end{gathered}
$$

existing whenever the series of type $(I I)$ terminates at zero term. This gives the following restrictions on the parameters

$$
\delta=\frac{1}{9}(\xi+2-\alpha-e)(2-3 \alpha+\xi), \sigma=\frac{1}{9}\left(12 \alpha+6 e+3 \xi \alpha-\xi^{2}-10 \xi-16\right) .
$$

Further, we set $e=3(27+6 \xi-4 \alpha) / 4$. If $k=0$ in relation (4.4), then it follows from Lemma 3.1 that $N=1$ and the irreducible invariant algebraic curve takes the form

$$
\begin{aligned}
F(x, y) & =y+x^{3}+\frac{3}{2} x^{2}-\left(\xi-\alpha+\frac{9}{2}\right) x-\frac{1}{3}\left(\sigma+\xi^{2}-\xi \alpha\right) \\
& -\frac{1}{4}(27+12 \xi-6 \alpha), \quad \lambda(x, y)=3 x-\xi-\frac{9}{2}
\end{aligned}
$$

provided that series $(I)$ terminates at zero term. This series terminates under the condition

$$
\delta=\frac{1}{24}(2 \xi+9)\left(18 \alpha+4 \xi \alpha-4 \sigma-4 \xi^{2}-36 \xi-81\right) .
$$


Now we consider the case $k=1$ and $N>1$. We calculate several first coefficients of the Puiseux series under consideration. Requiring that the non-polynomial part of the expression in brackets in (4.4) vanishes, we obtain necessary conditions for invariant algebraic curves to exist. Deriving the coefficients at $y^{N-1} x^{-l}, l \in \mathbb{N}$ in the representation of $F(x, y)$ in the field $\mathbb{C}_{\infty}\{x\}$ yields the following relations

$$
a_{l+2}+\sum_{j=1}^{N-1} c_{l+3}^{(j)}=0, \quad l \in \mathbb{N} .
$$

It is convenient to introduce the variables $C_{m}=\left\{c_{3}^{(1)}\right\}^{m}+\ldots+\left\{c_{3}^{(N-1)}\right\}^{m}$, where $m \in \mathbb{N}$. Setting $\xi=-2$, we use the algorithms of algebraic geometry, including the method of resultants and Gröbner bases, to solve this system. In practice we need the first fifteen equations given by (4.10) in order to find all other irreducible invariant algebraic curves. In particular, we obtain that $N=2$ and $N=3$. Note that there exist solutions with non-natural values of $N$. We exclude them. Sufficiency we verify by direct substitution into equation (2.3). The results are gathered in Table 1.

Concluding the proof let us mention that the upper bound on the degrees of irreducible invariant algebraic curves for Liénard dynamical system (4.2) with $\xi=-2$ is equal to 8 while the upper bound with respect to the variable $y$ equals 3 .

\section{Conclusion}

In this article we have derived an explicit expression for the cofactor related to an irreducible invariant algebraic curve of a polynomial dynamical systems in the plane. We have investigated uniqueness properties of irreducible invariant algebraic curves for planar polynomial dynamical systems. We have presented the general structure of irreducible invariant algebraic curves and their cofactors for Liénard dynamical systems with $\operatorname{deg} f<$ $\operatorname{deg} g<2 \operatorname{deg} f+1$. We have proved that Liénard dynamical systems in question can have at most two distinct irreducible invariant algebraic curves simultaneously. The method of the present article can be generalized to the case of non-autonomous dynamical systems in the plane [27] and higher dimensional dynamical systems [9].

\section{Acknowledgments}

The author would like to thank the reviewers for their helpful comments and suggestions.

\section{Appendix}

Let us describe a method, which can be used to perform the classification of Puiseux series satisfying an algebraic first-order ordinary differential equation $E\left(x, y, y_{x}\right)=0$. The lefthand side of this expression can be regarded as the sum of differential monomials given by

$$
M[y(x), x]=C x^{l} y^{j_{0}}\left\{\frac{d y}{d x}\right\}^{j_{1}}, \quad C \in \mathbb{C} \backslash\{0\}, \quad l, j_{0}, j_{1} \in \mathbb{N}_{0} .
$$


The set of all the differential monomials of the form (7.1) will be denoted as $\mathbb{M}$. In order to simplify notation the expression $W[x, y(x)]$ will stand for a polynomial in $x, y(x)$, and $y_{x}(x)$ with coefficients from the field $\mathbb{C}$.

Let us define the map $q: \mathbb{M} \rightarrow \mathbb{R}^{2}$ by the following rules

$$
C x^{q_{1}} y^{q_{2}} \mapsto q=\left(q_{1}, q_{2}\right), \quad \frac{d^{k} y}{d x^{k}} \mapsto q=(-k, 1), \quad q\left(M_{1} M_{2}\right)=q\left(M_{1}\right)+q\left(M_{2}\right),
$$

where $C \in \mathbb{C} \backslash\{0\}$ is a constant, $M_{1}$ and $M_{2}$ are differential monomials. We denote the set of all points $q \in \mathbb{R}^{2}$ corresponding to the differential monomials of equation $E\left(x, y, y_{x}\right)=0$ as $S(E)$. The convex hull of $S(E)$ is known as the Newton polygon of the equation under consideration.

The boundary of the Newton polygon consists of vertices and edges. Selecting all the differential monomials of the original equation that generate the vertices and the edges of the Newton polygon, we obtain a number of balances. The balance for a vertex is defined as the sum of those differential monomials in $E\left(x, y, y_{x}\right)$ that are mapped into the vertex. The balance for an edge is defined as the sum of differential monomials in $E\left(x, y, y_{x}\right)$ whose images belong to the edge. If solutions of the equation $E\left(x, y, y_{x}\right)=0$ possess an asymptotics of the form $y(x)=c_{0} x^{r}$ with $x \rightarrow 0$ or $x \rightarrow \infty$, then there exists a balance $W[x, y(x)]$ such that the function $y(x)=c_{0} x^{r}$ satisfies the equation $W[x, y(x)]=0$. Conversely, the function $y(x)=c_{0} x^{r}$ solving equation $W[x, y(x)]=0$, where $W[x, y(x)]$ is a balance, is an asymptotics at $x \rightarrow 0$ (or $x \rightarrow \infty$ ) for solutions of equation (2.4) whenever for all the differential monomials $M[x, y(x)]$ of the original equation not involved into $W[x, y(x)]$ we have $\operatorname{Re} \varkappa>\operatorname{Re} \varkappa_{0}\left(\right.$ or $\operatorname{Re} \varkappa<\operatorname{Re} \varkappa_{0}$ ), where $M\left[x, c_{0} x^{r}\right]=B x^{\varkappa}$ and $M_{0}\left[x, c_{0} x^{r}\right]=B_{0} x^{\varkappa_{0}}$ with $M_{0}[x, y(x)]$ being a differential monomial of the balance $W[x, y(x)]$.

Thus, having found all the power solutions $y(x)=c_{0} x^{r}$ for all the balances, one needs to select those that give asymptotics at $x \rightarrow 0$ or $x \rightarrow \infty$. Using power asymptotics it is possible to derive asymptotic series possessing these asymptotics as leading-order terms [22, 23]. In this article we are mainly interested in Puiseux series near $x=\infty$ that satisfy equation (2.4), therefore we shall focus at the case $r \in \mathbb{Q}$ and $x \rightarrow \infty$. Let us suppose that a balance $W[y(x), x]$ of the equation $E\left(x, y, y_{x}\right)=0$ has a solution $y(x)=c_{0} x^{r}$, which is an asymptotics at $x \rightarrow \infty$ and $r \in \mathbb{Q}$. In what follows we do not consider convergence of asymptotic Puiseux series. Note that the differentiation in the fields $\mathbb{C}_{x_{0}}\{x\}$ and $\mathbb{C}_{\infty}\{x\}$ is defined as a formal operation with most of the properties similar to those valid for convergent Puiseux series.

In order to obtain the structure of the corresponding series one should find the Gâteaux derivative of the balance $W[y(x), x]$ at the solution $y(x)=c_{0} x^{r}$ :

$$
\frac{\delta W}{\delta y}\left[c_{0} x^{r}\right]=\lim _{s \rightarrow 0} \frac{W\left[c_{0} x^{r}+s x^{r-p}, x\right]-W\left[c_{0} x^{r}, x\right]}{s}=V(p) x^{\tilde{r}} .
$$

In this expression $V(p)$ is a first-degree polynomial with respect to $p$. The coefficients of this polynomial depend on $c_{0}$ and on the parameters (if any) of the original equation involved into the balance $W[y(x), x]$. The zero $p_{0}$ of $V(p)$ is called the Fuchs index (or the resonance) of the balance $W[y(x), x]$ and its power solution $y(x)=c_{0} x^{r}$. Let $\operatorname{lcm}(n, m)$ be the lowest 
common multiple of two integer numbers $n$ and $m$. If the Fuchs index $p_{0}$ is not a positive rational number, then the number $n_{0}$ in expression (2.6) is given by $n_{0}=r_{2}$ where $r_{2}$ is defined as $r=r_{1} / r_{2}$ with $r_{1}$ and $r_{2}$ being coprime numbers, $r_{1} \in \mathbb{Z}$ and $r_{2} \in \mathbb{N}$. Otherwise we obtain $n_{0}=\operatorname{lcm}\left(g_{2}, r_{2}\right)$, where $r_{2}$ was defined previously and $g_{2}$ is given by $p_{0}=g_{1} / g_{2}$ with coprime natural numbers $g_{1}$ and $g_{2}$.

Finally, it is important to verify the existence of the Puiseux series of the form (2.6) with $l_{0}=r n_{0}$. If the balance $W[y(x), x]$ corresponds to a vertex of the Newton polygon, then the Puiseux series always exists and possesses an arbitrary coefficient $c_{0}$. In this case the Fuchs index is equal to zero. Now let us suppose that the balance $W[y(x), x]$ corresponds to an edge of the Newton polygon. Substituting series (2.6) into the equation $E\left(x, y, y_{x}\right)=0$ one can find the recurrence relation for its coefficients. This relation takes the form

$$
V\left(\frac{k}{n_{0}}\right) c_{k}=U_{k}\left(c_{0}, \ldots, c_{k-1}\right), \quad k \in \mathbb{N}
$$

where $U_{k}$ is a polynomial of its arguments. Note that $U_{k}$ can also depend on the parameters (if any) of the original equation. The equation $U_{n_{0} p_{0}}=0$ is called the compatibility condition. If the compatibility condition is not satisfied, then the Puiseux series under consideration does not exist. Otherwise the corresponding Puiseux series exists and possesses an arbitrary coefficient $c_{n_{0} p_{0}}$. Consequently, we conclude that the Puiseux series in question has uniquely determined coefficients provided that there are no non-negative rational Fuchs indices.

We note that if one wishes to find all the Puiseux series of the form (2.6) that satisfy the original equation, then it is necessary to implement the procedure described above for all the dominant balances and for all their power solutions $y(x)=c_{0} x^{r}$ with $r \in \mathbb{Q}$ and $x \rightarrow \infty$.

We also observe that there may exist balances and their power solutions such that $V(p) \equiv$ 0 . If $V(p)$ is identically zero, then one should make the substitution $y(x)=c_{0} x^{r}+w(x)$ in equation $E\left(x, y, y_{x}\right)=0$ and find all the Puiseux series $w(x)=c_{1} x^{r_{1}}+\ldots$ of the latter such that $r_{1}<r, r_{1} \in \mathbb{Q}$ and $x \rightarrow \infty$.

If one wishes to perform the classification of Puiseux series near finite non-zero points satisfying equation $E\left(x, y, y_{x}\right)=0$, then the following variables $w(s)=y\left(s+x_{0}\right)$ and $s=x-x_{0}$ should be introduced. Making this substitution yields another first-order algebraic ordinary differential equation. The method described above can be used to find balances near the point $s=0$ for the new equation. Some examples of this method in practice are given, for instance, in [23] and [28, 29].

\section{References}

[1] M. F. Singer, Liouvillian first integrals of differential systems, Trans. Amer. Math. Soc. 333 (1992) 673-688.

[2] C. J. Christopher, Invariant algebraic curves and conditions for a centre, Proc. Roy. Soc. Edinburgh Sect. A 124 (1994) 1209-1229.

[3] C. Christopher, Liouvillian first integrals of second order polynomial differential equations, Electron. J. Differential Equations 49 (1999) 1-7.

[4] Y. Ilyashenko, S. Yakovenko, Lectures on Analytic Differential Equations, Vol. 86, Graduate Studies in Mathematics, American Mathematical Society, 2008. 
[5] X. Zhang, Integrability of Dynamical Systems: Algebra and Analysis, Springer Singapore, 2017.

[6] J. Lei, On a classification of polynomial differential operators with respect to the type of first integrals, J. Differential Equations 260 (2016) 1993-2025.

[7] M. V. Demina, Novel algebraic aspects of Liouvillian integrability for two-dimensional polynomial dynamical systems, Physics Letters A 382 (20) (2018) 1353-1360.

[8] M. V. Demina, Invariant algebraic curves for Liénard dynamical systems revisited, Applied Mathematics Letters 84 (2018) 42-48.

[9] M. V. Demina, Classifying algebraic invariants and algebraically invariant solutions, Chaos, Solitons and Fractals 140 (2020) 110219.

[10] K. Odani, The limit cycle of the van der Pol equation is not algebraic, Journal of Differential Equations 115 (1) (1995) 146-152.

[11] H. Żołądec, Algebraic invariant curves for the Liénard equation, Trans. Amer. Math. Soc. 350 (1998) 1681-1701.

[12] J. Llibre, X. Zhang, On the algebraic limit cycles of Liénard systems, Nonlinearity 21 (2008) 2011-2022.

[13] J. Llibre, C. Valls, Liouvillian first integrals for Liénard polynomial differential systems, Proc. Amer. Math. Soc. 138 (9) (2010) 3229-3239.

[14] X. Yu, X. Zhang, The hyperelliptic limit cycles of the Liénard systems, Journal of Mathematical Analysis and Applications 376 (2011) 535-539.

[15] J. Giné, J. Llibre, Weierstrass integrability in Liénard differential systems, Journal of Mathematical Analysis and Applications 377 (2011) 362-369.

[16] C. Liu, G. Chen, J. Yang, On the hyperelliptic limit cycles of Liénard systems, Nonlinearity 25 (6) (2012) 1601-1611.

[17] J. Llibre, C. Valls, Liouvillian first integrals for generalized Liénard polynomial differential systems, Adv. Nonlinear Stud. 13 (2013) 819-829.

[18] G. Chèze, T. Cluzeau, On the nonexistence of Liouvillian first integrals for generalized Liénard polynomial differential systems, Journal of Nonlinear Mathematical Physics 20 (4) (2013) 475-479.

[19] A. O. Ignatyev, The domain of existence of a limit cycle of Liénard system, Lobachevskii Journal of Mathematics 38 (2) (2017) 271-279.

[20] M. V. Demina, C. Valls, On the Poincaré problem and Liouvillian integrability of quadratic Liénard differential equations, Proc. Roy. Soc. Edinburgh Sec. A: Mathematics 150 (6) (2020) 3231-3251.

[21] R. Walker, Algebraic Curves, Springer-Verlag, New York, 1978.

[22] A. D. Bruno, Asymptotic behaviour and expansions of solutions of an ordinary differential equation, Russ. Math. Surv. 59 (3) (2004) 429-481.

[23] A. D. Bruno, Power Geometry in Algebraic and Differential Equations, Elsevier Science (NorthHolland), 2000.

[24] R. Conte, The Painlevé approach to nonlinear ordinary differential equations, in: R. Conte (Ed.), The Painlevé Property One Century Later, CRM Series in Mathematical Physics, Springer-Verlag, New York, 1999, pp. 77-180.

[25] A. Goriely, Integrability and Nonintegrability of Dynamical Systems, World Scientific, 2001.

[26] A. Ferragut, H. Giacomini, A new algorithm for finding rational first integrals of polynomial vector fields, Qual. Theory Dyn. Syst. 9 (2010) 89-99.

[27] M. V. Demina, Invariant surfaces and Darboux integrability for non-autonomous dynamical systems in the plane, Journal of Physics A: Mathematical and Theoretical 51 (2018) 505202.

[28] M. V. Demina, N. A. Kudryashov, Power and non-power expansions of the solutions for the fourthorder analogue to the second Painlevé equation, Chaos, Solitons and Fractals 32 (1) (2007) 124-144.

[29] M. V. Demina, N. A. Kudryashov, The Yablonskii-Vorob'ev polynomials for the second Painlevé hierarchy, Chaos, Solitons and Fractals 32 (2) (2007) 526-537. 